\title{
Fundus autofluorescence imaging compared with different confocal scanning laser ophthalmoscopes
}

\author{
C Bellmann, G S Rubin, S A Kabanarou, A C Bird, F W Fitzke
}

Br J Ophthalmol 2003;87:1381-1386

See end of article for authors' affiliations

a.................

Correspondence to: Fred W Fitzke, PhD, Institute of Ophthalmology, Department of Visual Science, 11-43 Bath Street, London ECIV 9EL, UK; f.fitzke@ucl.ac.uk

Accepted for publication 4 February 2003

\begin{abstract}
Background: With the advent of confocal scanning laser ophthalmoscopes (cSLO), fundus autofluorescence (FAF) resulting mainly from lipofuscin accumulation on the level of the retinal pigment epithelium can be visualised in vivo. Various CSLOs are available to document FAF. The authors analysed and compared results of FAF using three different instruments.

Methods: Eight eyes of eight normal volunteers and 18 eyes of 12 patients with different retinal diseases (age related macular degeneration, macular dystrophy, central serous retinopathy) were examined. FAF images were recorded from each subject with the Heidelberg retina angiograph (HRA), the Rodenstock cSLO (RcSLO) and the Zeiss Prototype SM 30-4024 (ZcSLO). For excitation an argon laser (488 nm) was used (barrier filter: HRA $500 \mathrm{~nm}$; RcSLO $515 \mathrm{~nm}$; ZcSLO $521 \mathrm{~nm}$ ). $32 \mathrm{FAF}$ images were aligned and averaged using the same soffware for all cSLOs. FAF distribution was measured and grey scale values as well as root mean square (RMS) contrast were compared.

Results: Mean age of all subjects was 55.5 (SD 21.4) years. The maximum grey scale value averaged across all eyes was 76.19 (39.34) for the HRA, 61.44 (22.12) for the ZcSLO and 37.0 (9.97) for the RcSLO. The RMS contrast was $0.46(0.20)$ for the ZcSLO, $0.40(0.12)$ for the HRA, and $0.13(0.05)$ for the RcSLO. The differences between the cSLOs were statistically significant with higher grey scale levels and more contrast for the HRA and ZcSLO than the RcSLO (repeated measures ANOVA; $p<0.0001$ ). The differences between the HRA and the ZcSLO were not significant (post hoc comparisons; $p<0.05$ ).

Conclusions: All cSLOs allow clinically useful FAF imaging in retinal diseases. However, grey scale levels and contrast were much lower on the RcSLO. Therefore, RcSLO images appear much darker than HRA or ZcSLO images. Furthermore, not all cSLOs have a fixed photodetector gain and a standardised value for the argon laser amplification, which is mandatory for an absolute comparison of FAF imaging results.
\end{abstract}

ipofuscin (LF) accumulates with age within the lysosomal compartment of human postmitotic retinal pigment -epithelium (RPE) cells. Morphometric and in vitro fluorescence microscopic techniques have been used to obtain information about LF. ${ }^{1-7}$ These studies show that LF is derived from residual bodies that result from incomplete degradation of phagosomes, and may inhibit cellular RPE function. $^{8-11}$ Fundus spectrophotometric studies in vivo by Delori and co-workers, using a modified fundus camera, have shown that fundus autofluorescence (FAF) is mainly derived from RPE lipofuscin ${ }^{12-14}$ and is emitted over a large spectral band from $500-750 \mathrm{~nm}$ with its peak emission located between $620 \mathrm{~nm}$ and $640 \mathrm{~nm} .{ }^{12}$

FAF imaging has been developed and improved over the past decade and became a very important diagnostic tool in a variety of retinal diseases. ${ }^{15-29}$ FAF imaging allows visualising of the RPE as a single layer in vivo and may help to better understand its metabolic alterations in the pathogenesis of retinal disorders. In previous studies an increased FAF signal has been found in different hereditary disorders like Best macular dystrophy or Stargardt disease. ${ }^{14}{ }^{16} 25$ In age related macular degeneration (AMD) it has been shown that the FAF response is very low or extinguished in areas of atrophy but may be increased in the junctional zone around the areas of atrophy. ${ }^{19} 20$ An increased signal of FAF has been described not only in AMD patients with geographic atrophy, but also in patients with choroidal neovascularisation, drusen, and focal hyperpigmentations. ${ }^{16} 182224$ Therefore, FAF imaging may be not only a useful tool for documentation of retinal changes but also for identification of high risk characteristics in AMD. Furthermore, FAF imaging may be a useful technique to indicate new therapeutic interventions in AMD and to monitor their effect over time.

Various instruments are available to document FAF. In addition to the method used by Delori and co-workers, confocal scanning laser ophthalmoscopy (cSLO) is a useful technique for FAF imaging. It allows documentation of its spatial distribution over large retinal areas. ${ }^{16}{ }^{21}$ The aim of the study is not only to represent the distribution of FAF in normal eyes and in eyes with different retinal diseases, but also to analyse and compare FAF imaging results of three different cSLOs.

\section{MATERIAL AND METHODS}

For each subject FAF images were recorded with three different cSLOs (Heidelberg retina angiograph (HRA, Heidelberg Engineering, Dossenheim, Germany), Rodenstock cSLO (RcSLO, today Rodenstock, Weco, Düsseldorf, Germany) and the Zeiss prototype SM 304024 (ZcSLO, Zeiss, Oberkochen, Germany).

FAF images were produced using a $30^{\circ}$ field of view mode on the HRA. A $40^{\circ}$ field of view mode was chosen on the ZcSLO and on the RcSLO. In all cSLOs a confocal detection unit employs a small pinhole aperture to suppress light originating from outside the focal plane. This enhances image contrast when compared to a non-confocal image. The ametropic corrector is used to correct for refractive error and to focus on the structure of interest. For FAF imaging an argon blue laser with a wavelength of $488 \mathrm{~nm}$ is used for excitation on all cSLOs. Emitted light is detected above $500 \mathrm{~nm}$ for the HRA, above $515 \mathrm{~nm}$ for the RcSLO, and above $521 \mathrm{~nm}$ for the ZcSLO (barrier filter). In the HRA and the 
Table 1 Scanning laser ophthalmoscope characteristics

\begin{tabular}{llll}
\hline & HRA & Rodenstock SLO & Zeiss SLO \\
\hline Argon laser & $488 \mathrm{~nm}$ & $488 \mathrm{~nm}$ & $488 \mathrm{~nm}$ \\
Barrier filter & $500 \mathrm{~nm}$ & $515 \mathrm{~nm}$ & $521 \mathrm{~nm}$ \\
Field of view & $10^{\circ} / 20^{\circ} / 30^{\circ}$ & $20^{\circ} / 40^{\circ}$ & $20^{\circ} / 40^{\circ}$ \\
Laser output & $\approx 265 \mu \mathrm{W}$ & $\approx 255 \mu \mathrm{W}$ & $\approx 230 \mu \mathrm{W}$ \\
Detector & Photodiode & Photodiode & Photocathode \\
\hline
\end{tabular}

RcSLO a photodiode is used as a detector; on the ZcSLO a photocathode is employed. The argon laser output at the cornea surface was measured on all three cSLOs and was constant at $230 \mu \mathrm{W}$ for the HRA, at about $265 \mu \mathrm{W}$ for the ZcSLO, and at $255 \mu \mathrm{W}$ for the RcSLO. The image size for the HRA is $512 \times 512$ pixels, for the ZcSLO $768 \times 576$ pixels, and for the RCSLO $768 \times 576$ pixels (including the data field for patient and laser information). The characteristics of the cSLOs are summarised in Table 1.

FAF images were obtained in eight eyes of eight normal volunteers (mean age 33.5 (SD 7.05) years) and in 18 eyes of 12 patients (mean age 65.18 (14.41) years). Eleven eyes with $A M D$, six eyes with macular dystrophy and one eye with central serous retinopathy were examined. Before examination the pupil of the studied eye was dilated with phenylephrine $2.5 \%$ and tropicamide $1 \%$. Since all normal volunteers and patients had clear media, no correction for lens opacities was made.

All FAF images were consistently chosen to best image focus. For comparison of FAF images we decided to align and to calculate a mean of a sequence of 32 single images as it has been described for previous studies. ${ }^{23}$ The same image analysis software was used for all cSLOs. Topographic distribution and intensity of FAF throughout the posterior pole was determined by measuring grey scale values (Matrox inspector, Matrox Electronic Systems Ltd, Québec, Canada)
(Figs 1 and 2). Grey scale levels were obtained from a horizontal single pixel line over the whole image width up to 751 pixels from the optic nerve head to the temporal aspect of the macula through the fovea. For image brightness the maximum grey scale value was calculated and averaged across all eyes. Image contrast was measured as root mean square (RMS)-contrast which is defined as the standard deviation divided by the mean of grey scale values. Therefore, the RMS-contrast is a normalised value between 0 and $1 .{ }^{30}$

Artificial eye measurements were performed using a composite of a fluorescence standard (F-2225, Avian Technologies LLC, OH, USA), an optical lens and a neutral density optical filter $0.5 \log$ units ND. Additional measurements were made after removal of the fluorescence standard and choosing a black background to determine the background noise. For both measurements (with and without the fluorescence standard) the same settings on each machine were chosen. For the RCSLO this was the maximum value for the photodetector gain and for the ZcSLO a standardised value which has been determined already for previous studies. ${ }^{23}$ The photodetector gain of the HRA was variable and a setting was determined, which was comparable with FAF imaging in patients. Image recording as well as further image analyses were performed in the same way as described above for FAF images obtained in vivo. Additionally, the range of grey scale values was calculated as the difference of

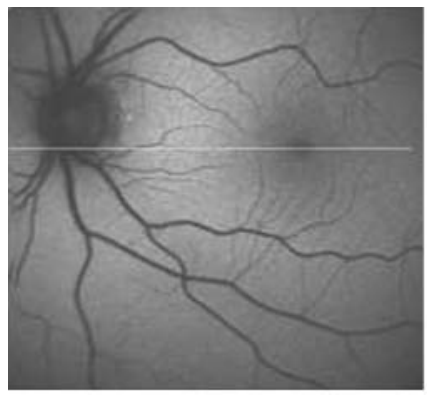

A

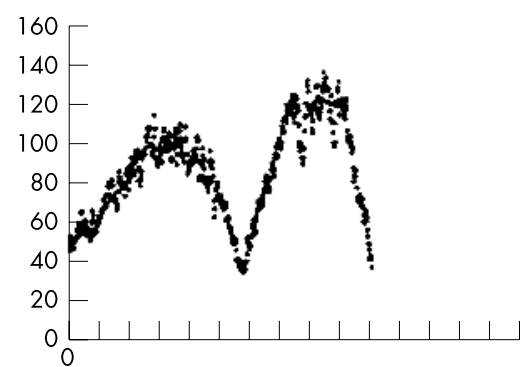

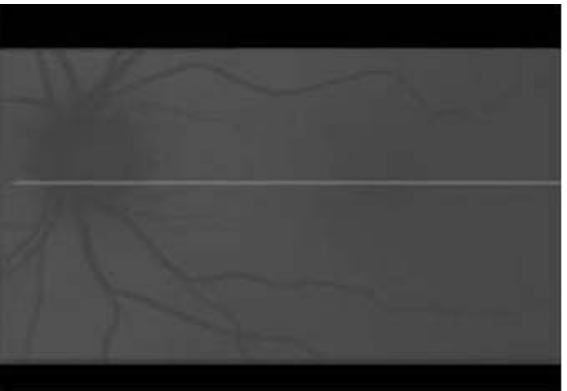

B

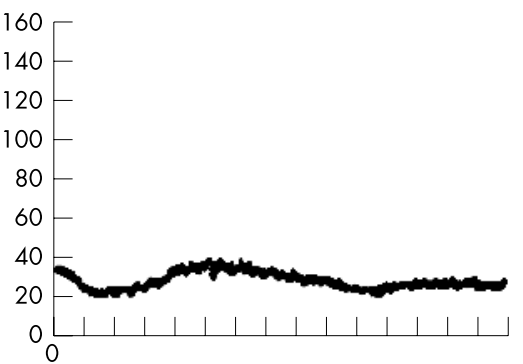

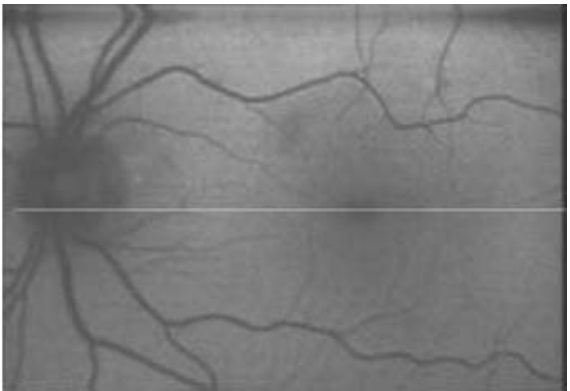

C

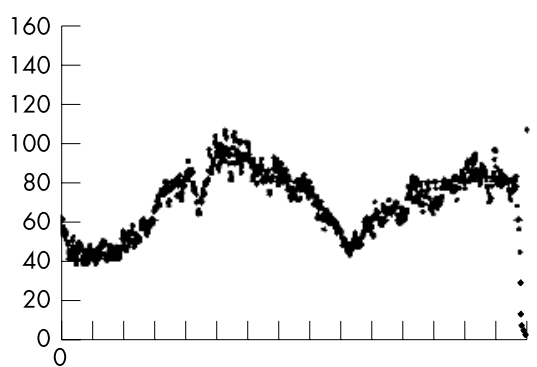

Figure 1 Fundus autofluorescence (FAF) images and corresponding grey scale values in a normal subject. Grey scale values were highest on the Heidelberg retina angiograph (A) and Zeiss cSLO (C), lowest on the Rodenstock cSLO (B). A one pixel line is placed from the temporal aspect of the macula through the fovea to the optic nerve head. The FAF signal is lower in the fovea because of blue light absorption of the macular luteal pigment. The FAF signal is absent over retinal vessels and the optic nerve head. Highest grey scale values are obtained in the parafoveal region on all 3 cSLOs. 


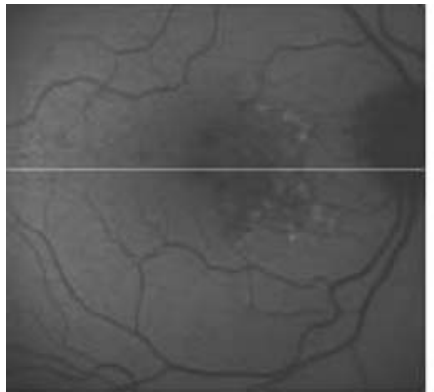

A

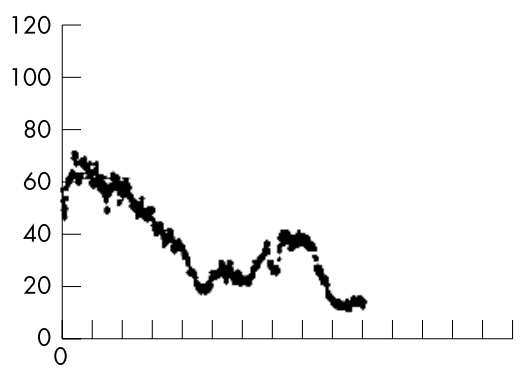

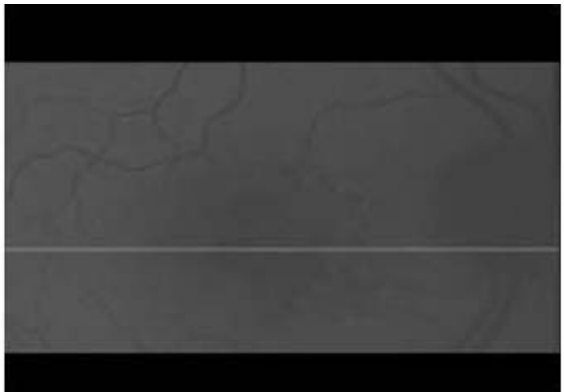

B

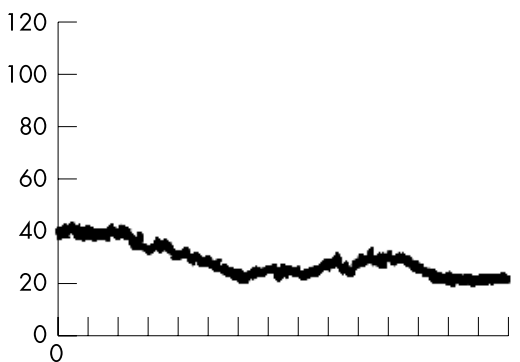

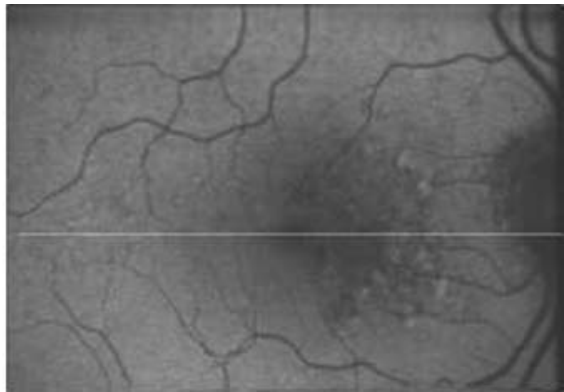

C

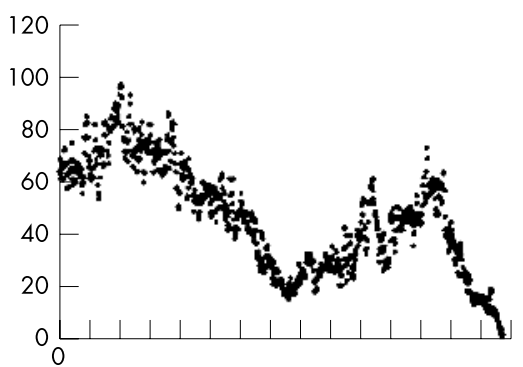

Figure 2 Fundus auofluorescence images and corresponding grey scale levels in a patient with macular dystrophy. Grey scale values were highest on the Heidelberg retina angiograph $(A)$ and Zeiss cSLO (C), and lowest on the Rodenstock cSLO (B). Although the Zeiss cSLO (C) image appears noisier in comparison with the HRA image (A) the image contrast between the ZcSLO and the HRA is similar.

grey scale values measured with the fluorescence standard and after its removal using a black background for all cSLOs (Fig 3) (Table 2).

The tenets of the Declaration of Helsinki were followed and the study has been approved by the local ethics committee.

\section{RESULTS}

According to the measured grey scale values the brightest images were obtained with the HRA. The RcSLO produces the darkest images in normal subjects as well as in patients. The maximum grey scale values averaged across all eyes were 71.13 (SD 35.24) for the HRA, 67.63 (10.14) for the ZcSLO, and 38.88 (12.64) for the RcSLO in normal subjects. The grey scale distribution was similar in patients. The means of maximum grey scale values were 78.32 (28.68) for the HRA, 59.11 (21.58) for the ZcSLO, and 36.21 (8.89) for the RcSLO (Fig 4). Separated repeated measures (ANOVA) showed significant differences between the cSLOs with $\mathrm{p}=0.02$ for normal volunteers and with $\mathrm{p}=0.0003$ for patients. Post hoc comparisons indicated that grey scale values were significantly higher $(\mathrm{p}<0.05$, Tukey/Kramer $)$ for both, the HRA and the ZcSLO than for the RCSLO in normal subjects and in patients. The difference in maximum grey scale values between the HRA and the ZcSLO was not significant.

Additionally, in normal subjects and in patients we measured the RMS-contrast on each image separately. The RMS-contrast showed highest values on ZcSLO and HRA images and lowest on RcSLO images (Fig 5). In normal subjects the RMS-contrast averaged across all eyes was 0.41
(0.21) for the ZcSLO, 0.39 (0.05) for the HRA, and 0.15 (0.02) for the RCSLO. The values of FAF images in patients were similar (ZcSLO 0.48 (0.19), HRA 0.40 (0.15), RcSLO 0.13 $(0.06))$. The difference between the cSLOs was significant in both groups (ANOVA repeated measures with $\mathrm{p}=0.002$ for normal subjects and $\mathrm{p}<0.0001$ for patients). Consecutive post hoc comparison indicated a significant difference between the HRA and ZcSLO in comparison with the RcSLO ( $p<0.05$; Tukey/Kramer). We found no significant difference in contrast between the HRA and ZcSLO on FAF images obtained in normal subjects and in patients.

Similarly, grey scale levels were determined using the artificial eye. Measurements with the fluorescence standard showed highest grey scale values for the HRA and lowest for the RcSLO (Table 2). The mean of grey scale values was 169.68 (43.96) for the HRA, 159.19 (22.34) for the ZcSLO, and 60.89 (12.76) for the RcSLO. For the background noise lowest results were obtained on the HRA (mean 11.21 (1.33)). For the RcSLO we measured a mean grey scale level of $25.45(0.65)$ and for the ZcSLO a mean value of 54.02 (5.58). The range of values was highest for the HRA and lowest for the RcSLO (HRA mean 158.46 (44.01), ZcSLO 105.17 (19.62), RcSLO 35.43 (12.82)) (Table 2).

\section{DISCUSSION}

Although FAF imaging has been of growing interest as a diagnostic tool in retinal diseases now for several years, image quality and differences between the instruments used have not been described before.

Table 2 Grey scale values and range of grey scale values (mean (SD))

\begin{tabular}{llcr}
\hline & \multicolumn{2}{l}{ Grey scale values over whole image width } & \\
\cline { 2 - 3 } & Fluorescence standard & Background noise & Range of grey scale values \\
\hline HRA & $169.68(43.96)$ & $11.21(1.33)$ & $158.46(44.01)$ \\
Rodenstock cSLO & $60.89(12.76)$ & $25.45(0.65)$ & $35.43(12.82)$ \\
Zeiss cSLO & $159.19(22.34)$ & $54.02(5.58)$ & $105.17(19.62)$ \\
\hline
\end{tabular}


A

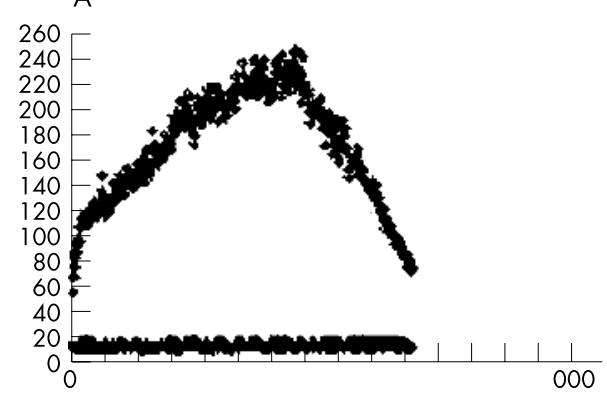

B

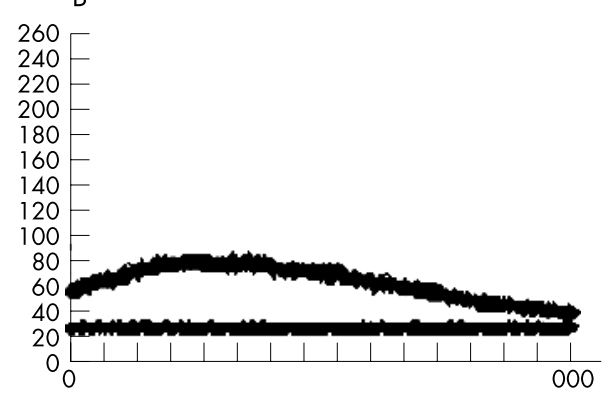

C

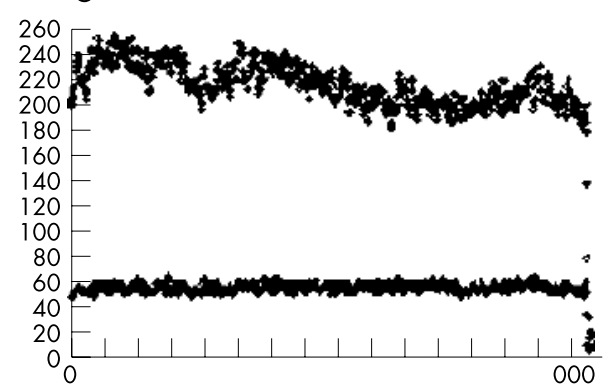

Figure 3 Artificial eye measurements using a fluorescence standard (upper line) and after its removal (background noise) (lower line): Heidelberg retina angiograph (HRA) (A), Rodenstock cSLO (B), and the Zeiss CSLO (C). After averaging 32 images grey scale values were measured over the whole image width. Fluorescence standard measurements have shown highest grey scale values on the HRA (A) and lowest on the Rodenstock cSLO (B). Background noise measurements have shown lowest results on the HRA (A) and highest on the Zeiss CSLO (C). The range of grey scale values was highest on the HRA (A) and lowest on the Rodenstock cSLO (B). The highest light dropoff with lowest grey scale values in the image periphery was found on the HRA (A).
To obtain FAF images in vivo Delori et al used a modified fundus camera coupled to a scientific grade CCD camera. $^{12-14} 18$ Two different excitation filters (467 nm and $550 \mathrm{~nm}$ ) and barrier filters (499 nm and $576 \mathrm{~nm}$ ) allowed FAF imaging at two different wavelengths. An aperture, which was inserted in the optical pathway, allowed an image size of about 13 degrees in diameter. However, to correlate fundus changes with FAF results a cSLO is a very useful instrument and is widely used today for FAF imaging. ${ }^{15-29}$ An image size up to 40 degrees in diameter, depending on the choice of the cSLO, is available (Table 1).

We compared the FAF image quality using three different cSLOs which were available at the Institute of Ophthalmology and Moorfields Eye Hospital, London. We chose a technique which allows measuring grey scale values over the whole image diameter. Grey scale values provide information about image brightness and contrast. Although visual image inspection may show further differences, brightness and contrast represent important indicators for image quality. We recorded significant differences in image quality between the cSLOs. Highest grey scale levels were measured and therefore brightest FAF images were obtained on the HRA and the ZcSLO, not only in normal subjects but also in patients with a variety of retinal disorders. Lowest values and therefore darkest FAF images were found on the RCSLO. Furthermore, the RMS-contrast was much lower on RCSLO images and explains why details are less visible on RcSLO image in comparison with ZcSLO and HRA images (see Fig 2). FAF image quality may be influenced by optical changes or by different scan angles between the cSLO and the patient's eye. ${ }^{31}$ To exclude these factors additional measurements were undertaken by using an artificial eye. By using this technique we obtained highest grey scale values on the HRA and lowest on the RcSLO whereas the background noise was highest on the ZcSLO and lowest on the HRA. The high background noise on the ZcSLO explains why the in vivo recordings on the ZcSLO appear much noisier in comparison with the HRA and RCSLO images (see Figs 1 and 2). Moreover, the differences in the range of grey scale values obtained by using the artificial eye support the finding that the RcSLO images appear much darker than HRA and ZcSLO images.

We compared technical details of all cSLOs. On all instruments a non-modified argon laser with a wavelength of $488 \mathrm{~nm}$ was used for excitation. The laser output was constant and comparable on all three cSLOs with $230 \mu \mathrm{W}$ for the ZcSLO, $255 \mu \mathrm{W}$ for the RcSLO, and $265 \mu \mathrm{W}$ for the HRA. Since the laser output of the RcSLO was higher than of the ZcSLO and lower than of the HRA it is unlikely that the laser power accounts for the differences in image quality we obtained by comparing the recordings of all three cSLOs. It is more likely that differences in the optical pathway of the devices may explain why more contrast and brightness was
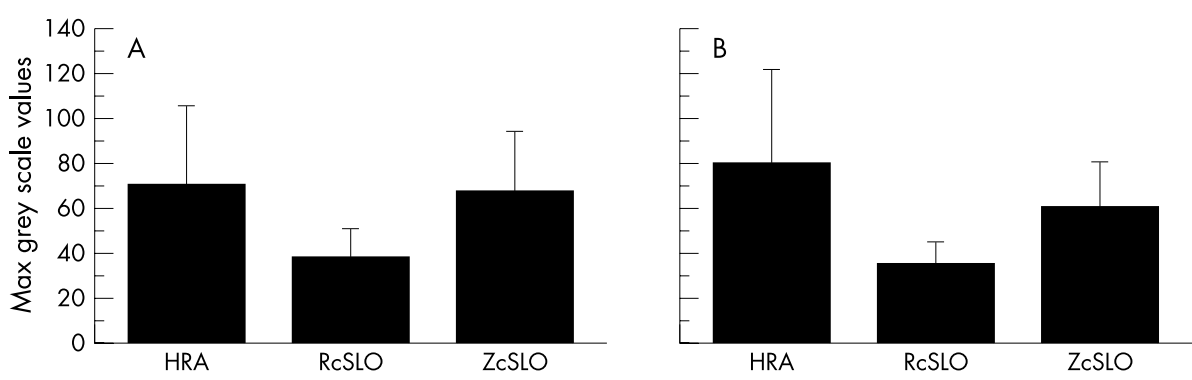

Figure 4 Maximum grey scale levels on the Heidelberg retina angiograph (HRA), Rodenstock confocal scanning laser ophthalmoscope (RcSLO) and Zeiss confocal scanning laser ophthalmoscope (ZcSLO). Repeated measures (ANOVA) showed significant difference between the devices (normal subjects $p=0.02$, patients $p=0.0003$ ). Post hoc comparisons indicated that grey scale values were significantly higher for the HRA and ZcSLO than for the RcSLO for both, normal subjects (A) and patients (B) $(p<0.05$, Tukey) Kramer). The difference between the HRA and ZcSLO was not significant. 


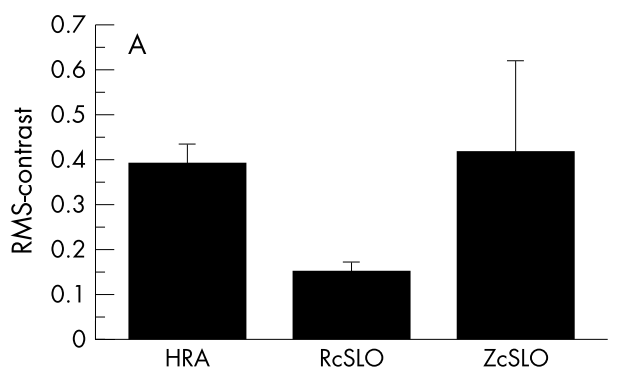

RcSLO

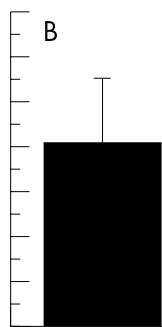

HRA

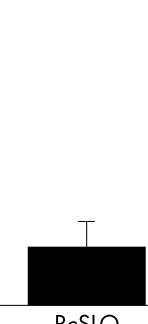

RcSLO

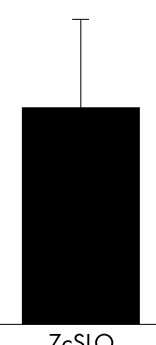

ZcSLO
Figure 5 RMS-contrast of Heidelberg retina angiograph (HRA) images, Rodenstock confocal scanning laser ophthalmoscope (RcSLO) images, and Zeiss confocal scanning laser ophthalmoscope (ZcSLO) images. Repeated measures (ANOVA) showed significant difference between the devices (normal subjects $p=0.002$, patients $p<0.0001$ ). Post hoc comparisons indicated that the RMScontrast were significantly higher on HRA and ZcSLO images than on RcSLO images for normal subjects (A) and for patients (B) ( $p<0.05$, Tukey/Kramer). The difference between the HRA and ZcSLO was not significant. obtained on HRA and ZcSLO images. The wavelength of the barrier filter was different on all three cSLOs. LF autofluorescence has a relatively broad emission spectrum with a peak value located at 620-640 nm. ${ }^{12}$ Hence, except for only a small fraction of light blocked at a shorter wavelength, most of the emitted light passes the barrier filter at $500 \mathrm{~nm}$ on the HRA, at $515 \mathrm{~nm}$ on the RcSLO, and at $521 \mathrm{~nm}$ on the ZcSLO. ${ }^{16} 192329$ However, the cut-off filter of the RcSLO is at a longer wavelength in comparison with the filter employed on the HRA and at a shorter wavelength compared with the filter incorporated on the ZcSLO. Therefore, factors other than laser output and the amount of light blocked by the filter must influence image quality. These may include the size of the pinhole aperture, the degree of amplification of the argon laser as well as the sensitivity of the incorporated photodetector.

On the HRA the photodetector gain is variable. Therefore, for artificial eye measurements we determined a setting which was comparable with measurements in patients. For the ZcSLO a fixed setting was used for all measurements which was determined in previous studies. ${ }^{23}$ For the RcSLO the maximum value was chosen for measurements in patients as well as for measurements using the artificial eye. The amplification of the argon laser may be variable as well which makes an absolute comparison of FAF results difficult. A specified setting with standardised values for the argon laser amplification as well as for the photodetector gain is mandatory not only to compare the results between different cSLOs but also between patients and examination over time on the same device.

Single FAF images are noisier and details are less visible than on an averaged image sequence. ${ }^{19}$ It is therefore important, to choose the same length of image sequence on each patient to compare FAF results. We decided to align and to calculate a mean of a sequence of 32 single images as it has been described before. ${ }^{23}$ The necessary program to calculate the mean image was the same for all three devices which allows us to exclude influences on image quality caused by the employed software.

In summary, all cSLOs allow clinically useful FAF imaging. However, our measurements have shown significant differences in image brightness and contrast as well as in the range of grey scale values as important indicators for image quality. Not all cSLOs have a fixed photodetector gain and a standardised value for the argon laser amplification which is mandatory for an absolute comparison of FAF imaging results. Furthermore, the number of averaged images and the software used for consecutive image analysis may have an influence on image quality. This has to be taken in account and carefully considered using FAF imaging as a diagnostic tool in retinal diseases especially with regard to comparison of FAF results between patients and over time.

\section{Authors' affiliations}

C Bellmann, G S Rubin, S A Kabanarou, A C Bird, F W Fitzke, Institute of Ophthalmology, University College London, UK C Bellmann, S A Kabanarou, A C Bird, Moorfields Eye Hospital, London, UK

Caren Bellmann is a Marie Curie Individual Fellow at the Institute of Ophthalmology (Marie Curie Individual Fellowship, European Commission No QLK6-CT2000-51262). Presented in part at the ARVO Meeting 2002, Fort Lauderdale, USA.

\section{REFERENCES}

1 Dorey KC, Wu G, Ebenstein D, et al. Cell loss in aging retina. Relationship to lipofuscin accumulation and macular degeneration. Invest Ophthalmol Vis Sci 1989;30:1691-19.

2 Feeney L. Lipofuscin and melanin of human retinal pigment epithelium. Fluorescence, enzyme cytochemical, and ultrastructure studies. Invest Ophthalmol Vis Sci 1978;17:583-600.

3 Feeney-Burns L, Berman ER, Rothman H. Lipofuscin of human retinal pigment epithelium. Am J Ophthalmol 1980;90:783-91.

4 Feeney-Burns L, Eldred GE. The fate of the phagosome: conversion to "age pigment" and impact in the human retinal pigment epithelium. Trans Ophthalmol Soc UK 1983;103:416-21.

5 Feeney-Burns L, Hilderbrand ES, Eldridge S. Aging human RPE: morphometric analysis of macular, equatorial, and peripheral cells. Invest Ophthalmol Vis Sci 1984;25:195-200.

6 Weiter JJ, Delori FC, Wing GL, et al. Retinal pigment epithelial lipofuscin and melanin and choroidal melanin in human eyes. Invest Ophthalmol Vis Sci 1986;27:145-52.

7 Wing GL, Blanchard GC, Weiter JJ. The topography and age relationship of lipofuscin concentration in the retinal pigment epithelium. Invest Ophthalmol Vis Sci 1978;17:601-7.

8 Boulton M, McKechnie M, Breda J, et al. The formation of autofluorescent granules in cultured human RPE. Invest Ophthalmol Vis Sci 1989:30:82-9.

9 Katz ML, Gao CL, Rice LM. Formation of lipofuscin like-fluorophores by reaction of the retinal with photoreceptor outer segements and liposomes. Mech Ageing Dev 1996;92:159-74.

10 Kennedy CJ, Rakocy PE, Constable IJ. Lipofuscin of the retinal pigment epithelium: a review. Eye 1995:9:763-71.

11 Holz FG, Schütt F, Kopitz J, et al. Inhibition of lysosomal degradative functions in RPE cells by a retinoid of component of lipofuscin. Invest Ophthalmol Vis Sci 1999:40:737-43.

12 Delori FC, Dorey CK, Staurenghi G, et al. In vivo fluorescence of the ocular fundus exhibits retinal pigment epithelium lipofuscin characteristics. Invest Ophthalmol Vis Sci 1995;36:718-29.

13 Delori FC, Goger DG, Dorey KC. Age-related accumulation and spatial distribution of lipofuscin in RPE of normal subjects. Invest Ophthalmol Vis Sci 2001;42:1855-66.

14 Delori FC, Staurenghi G, Arend O, et al. In vivo measurement of lipofuscin in Stargardt's disease-fundus flavimaculatus. Invest Ophthalmol Vis Sci 1995; $36: 2327-31$

15 Bellmann C, Holz FG, Breitbart A, et al. Bilaterale akute syphilitische posteriore plakoide chorioretinopathie (ASPPC). Angiographie- und Autofluoreszenzmerkmale. Ophthalmologe 1999;96:522-8.

16 Bellmann C, Holz FG, Schapp O, et al. Topographie der Fundusautofluoreszenz bestimmt mit einem konfokalen Scanning-LaserOphthalmoskop. Ophthalmologe 1997;94:385-91.

17 Bellmann C, Jorzik J, Spital G, et al. Symmetric manifestation of geographic atrophy of the retinal pigment epithelium in patients with age related macular degeneration. Arch Ophthalmol 2002; 120:579-84.

18 Delori FC, Fleckner MR, Goger DG, et al. Autofluorescence distribution associated with drusen in age-related macular degeneration. Invest Ophthalmol Vis Sci 2000;41:496-504.

19 Holz FG, Bellmann C, Margaritidis M, et al. Patterns of increased in vivo fundus autofluorescence in the junctional zone of geographic atrophy of the 
retinal pigment epithelium associated with age-related macular degeneration. Graefes Arch Exp Clin Ophthalmol 1999;237:145-52.

20 Holz FG, Bellmann C, Staudt S, et al. Fundus autofluorescence and development of geographic atrophy in age-related macular degeneration. Invest Ophthalmol Vis Sci 2001:42:1051-6.

21 Lois N, Halfyard AS, Bird AC, et al. Quantitative evaluation of fundus autofluorescence imaged in vivo in eyes with retinal disease. Br J Ophthalmol 2000;84:741-5.

22 Lois N Owens SL, Coco R, et al. Fundus autofluorescence in patients with agerelated macular degeneration and high risk of visual loss. Am J Ophthalmol 2002;133:341-9.

23 Rückmann A, Fitzke FW, Bird AC. Distribution of fundus autofluorescence with a scanning laser ophthalmoscope. Br J Ophthalmol 1995;79:407-12.

24 Rückmann A, Fitzke FW, Bird AC. Fundus autofluorescence in age-related macular disease imaged with a laser scanning ophthalmoscope. Invest Ophthalmol Vis Sci 1997;38:478-86.
25 Rückmann A, Fitzke FW, Bird AC. In vivo fundus autofluorescence in macular dystrophies. Arch Ophthalmol 1997;115:609-15.

26 Rückmann A, Fitzke FW, Fan J, et al. Abnormalities of fundus autofluorescence in central serous retinopathy. Am J Ophthalmol 2002;133:780-6.

27 Solbach U, Keilhaver $\mathrm{C}$, Knabben $\mathrm{H}$, et al. Imaging of retinal autofluorescence in patients with age-related macular degeneration. Retina 1997;17:385-9.

28 Spital G, Radermacher M, Müller C, et al. Autofluorescence characteristics of lipofuscin components in different forms of late senile macular degeneration. Klin Monatsbl Augenheilkd 1998;213:23-31.

29 Sunness JS, Ziegler M, Fujii G, et al. The use of SLO autofluorescence imaging to define and quantify atrophy in macular disease. Invest Ophthalmol 2000;41:S595.

30 Peli E. Contrast in complex images. J Opt Soc Am A 1990:7:2032-40.

31 Arnold JV, Gates JWC, Taylor KM. Possible errors in the measurement of retinal lesions. Invest Ophthalmol Vis Sci 1993;34:2576-80.

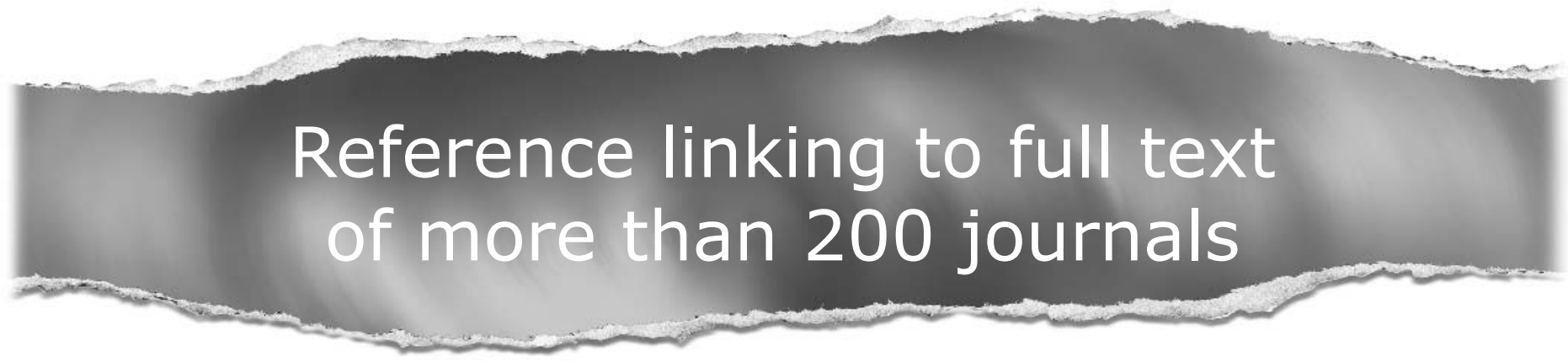

\section{Toll free links}

You can access the FULL TEXT of articles cited in the British Journal of Ophthalmology online if the citation is to one of the more than 200 journals hosted by HighWire (http://highwire.stanford.edu) without a subscription to that journal. There are also direct links from references to the Medline abstract for other titles.

\section{www.bjophthalmol.com}

